\title{
THE STABILITY OF FENARIMOL, TRIFLUMIZOLE AND PENCONAZOLE IN THEIR FORMULATIONS UNDER CERTAIN ENVIRONMENTAL CONDITIONS
}

\author{
ELBADRY, B. ELS. M. AND OLA M.Y. EMARA
}

Pesticides Analysis Res. Div., Central Agric.Pesticides Lab., ARC, Dokki, Giza

(Manuscript received 13 January 2009)

\begin{abstract}
The present work was carried out to study the persistence of Fenarimol (Rubigan $12 \% \mathrm{EC}$ ), Triflumizole (Trifimine 15\%EC) and Penconazole (Topas $10 \% \mathrm{EC}$ ) under certain environmental factors. The stability of active ingredients under accelerated storage through $54^{\circ} \mathrm{C}$ for $14-60$ days, and through different temperatures $\left(25^{\circ}, 35^{\circ} \mathrm{C}\right.$ and $\left.45^{\circ} \mathrm{C}\right)$ for $1-192$ hours, under direct ultraviolet and sunlight rays, were also considered. Generally the chemical residues of the studied pesticides were estimated by HPLC. The obtained results proved that : Fenarimol was more stable than other pesticides after storage for different period at $54^{\circ} \mathrm{C}$ and effect different temperature $\left(25^{\circ}, 35^{\circ} \mathrm{C}\right.$ and $\left.45^{\circ} \mathrm{C}\right)$. On the other hand, under direct ultraviolet and direct sunlight rays. The data presented showed that the rate of degradation of Penconazole was more rapid than Fenariol and Triflumizole in their formulations, while Triflumizole was more stable. Finally the different physical properties for unstored and stored tested pesticides are all within the allowed limits according to WHO and FAO.
\end{abstract}

\section{INTRODUCTION}

The increasing importance of environmental aspects in agriculture involved the production of chemicals that will assure the best crop production. These products must be highly effective but not toxic to humans, easily decomposed and at low environmental impact. In environmental field, partial degradation with parallel and subsequent degradation happens and might residues undesirable toxic substances(Samtoro et al 2000). Azoles (traizole, imidazole and pyrimidine) derivatives represent the most important category of fungicides to date. Thanks to their excellent protective, curative and eradicate power against a wide-spectrum of crop diseases. Triazole fungicides have a common structure moiety, the 1,2,4-triazole ring, which is connected to hydrophobic backbone thought position 1. Typically, the hydrocarbon backbone has a substituted phenyl group at one end, and an alkyl group or a different substituted phenyl group at the other end. As a consequence, a symmetrical carbons are generally present at the position(s) immediate and/or vicinal to the Ttriazole rings. This makes chirality's almost ubiquitous for Triazole-type Fungicides( Attenburger (1995) and Tomlin (1994)). 
O-toluidine derivative and propoxyethylidine, which are connected to imidazol-1yl group is attached directly through its-2- position have pestcidial activity, we have now found that the other compounds comprising an imidazole substituted in the -2positions directly or indirectly by aryl-substituted azolyl groups have good pesticidal activity. Triflumizole and its metabolites containing the 4-chloro-2trifluoromethylamiline moiety. Triflumizole, is a fungicide which controls a wide range of diseases in many economically important crops. Fenarimol is an extensively used fungicide which is composed of two chlorobenzene and one pyrimidine rings, connected by carbinol group. Whilst it has long been known that this compound readily breaking down by sunlight there is little published information on its photo stability under environmental conditions. In a previous report . we presented a spectroscopic study of the fungicide, showing that the lowest excited singlet state, corresponding to absorption between 280 and $320 \mathrm{~nm}$, is localized on the pyrimidine ring and has $n, \pi^{*}$ character. This overlaps with solar emission spectrum is expected to play a dominant role in the photo degradation process of this fungicide under natural environmental conditions (Mateus et al., 2000 and Samtoro et al 2000). The aim of this study is to obtain more information about the persistence of Fenarimol, Triflumizole and Penconazole in their formulations under certain environmental conditions such as storage for different periods and the effect of exposure to three different temperatures $\left(25^{\circ} \mathrm{C}, 35^{\circ} \mathrm{C}\right.$ and $\left.45^{\circ} \mathrm{C}\right)$. Finally ultraviolet and sunlight. Also the present work was directed to study the different physical properties, of tested pesticides formulations as affected by the storage temperature $\left(54^{\circ} \mathrm{C}\right)$ for different periods .

\section{MATERIALS AND METHODS}

\section{Pesticides under test}

\section{a) Fenarimol}

Common Name: Fenarimol

IUPAC Name: ( \pm )-2,4-dichloro- $\alpha$ (pyrimidin-5-yl) benzhydryl alchol.

\section{Chemical structure}<smiles>OC(c1ccc(Cl)cc1)(c1cncnc1)c1ccccc1Cl</smiles>

Trade Name: Rubigan 12\% EC

b) Triflumizole 
Common Name: Triflumizole

IUPAC Name: (E)-4-chloro- $\alpha, \alpha, \alpha$-trifluoro-N-(1-imidazol-1-yl-2propoxy-ethylidene)-otoluidine.

Chemical structure:<smiles>CCOCC(=Nc1ccc(Cl)cc1C(F)(F)F)n1ccnc1</smiles>

Trade Name: Trifimine 15\% EC

c) Penconazole

Common Name: Penconazole

IUPAC Name: 1 -(2,4-dichloro- $\beta$-propyl phenethyl) $-1 \mathrm{H}-1,2,4$ triazole.

Chemical structure:<smiles>CCCCCC(C)c1ccc(Cl)cc1Cl</smiles>

Trade Name: Topas $10 \%$ EC

EC: Emulsifiable Concentration

II. Effect of certain environmental conditions on the fate of Finarimol, Triflumizole and Penconazole formulations

1. Storage stability at elevated temperature

Storage at $54^{\circ} \mathrm{C} \pm 2$ for 14 days (FAO/WHO Manual 2002 and 2006), FAO specifications (1988). Then completed to 60 days (FAO/ WHO Manual 2002).

\section{Exposure as thin dry film on glass surfaces}

\section{General Method}

$1 \mathrm{ml}$ aliquots of the tested pesticides solutions in acetone containing $\mathbf{5 0 0} \mu \mathrm{g}$ a.i ( active ingredient) each spread as uniformly as possible on the surface of uncovered Petri dishes $(5 \mathrm{~cm}$ i.d). The acetone solvent was left to dry at room temperature. 


\subsection{Effect of different temperatures}

Exposed to 25,35 and $45^{\circ} \mathrm{C}$ for zero- 192 hours inside a dark electric oven provided with temperature regulation system.

\subsection{Effect ultraviolet rays (UV-rays) and direct sunlight}

Exposed to short wave length of uv-rays $(254 \mathrm{~nm})$ at a distance of $12 \mathrm{~cm}$ for 0 , $1,2,4,8$ hours.

Exposed to direct sunlight for $0,1,2,4,6,8,12,24,48$ and 72 hours. Dominating temperature was ranged between 32 and $38^{\circ} \mathrm{C}$.

Residues of tested pesticides from steps $(2,3)$ which were remained on exposed surface were quantitavely transferred to standard glass stopper test tubes with dichloromethane and the solvent was evaporated to dryness and the residues were determined by HPLC.

\section{Chemical Analysis}

Determination of active ingredient of Finarimol, Triflumizole and Penconazole as follow

- Estimate of (a.i) present before and after storage, and estimate of residues of tested pesticides on glass surface by HPLC instrument .

\section{High Pressure Liquid Chromatography (HPLC) Conditions}

The chromatographic system consisted of Jusco HPLC, diodearry detector Model 12015 and intelligent quaternary pump Model PU. 2089. Using $\mathrm{C}_{18}$ stainless steel column $\left(25 \mathrm{~cm} \times 4.6 \mathrm{~mm}\right.$ i.d) at $40^{\circ} \mathrm{C}$. Fenarimol, Triflumizole and Penconazole in their formulation were eluted isocratically by Methanol -Acetonitril-Water (70:25:5), at the rate of $1 \mathrm{Im} / \mathrm{min}$ and wave length235 and $254 \mathrm{~nm}$ respectively. Under these conditions. The $\mathbf{R}_{\mathbf{t}}$ were 3.8, 3.9and $4.9 \mathrm{~min}$ for tested pesticides, respectively. The results of tested insecticides were quanitively determined by comparison with standard solutions injected under the identical HPLC conditions according to the methods of ( Morse lab (1989)with some adaptation.

IV. Physical properties were evaluated before and after different storage periods in this study:-

\section{a)Density properties}

b) Emulsion

c) Free Acidity or Alkalinity

d) $\mathrm{pH}$ values
MT 3.2 CIPAC F( 1995)

MT 36 CIPAC F(1995)

MT 31 CIPACF(1995)

MT 75 CIPAC F (1995)

V. Kinetic Study: The half life periods $\boldsymbol{R}_{\boldsymbol{5 0}}$ for tested pesticides were estimated according to equation (Moye et al 1987).

$R L_{50}=\ln 2 / k=0.6932 / k$

where $\boldsymbol{k}$. rate of decomposition

$\mathbf{t}_{\mathbf{x}}$ : time of residue detection. $k=1 / t_{x}$. In $a / b_{x}$

, a: initial residue.

$\mathbf{b}_{\mathbf{x}}$ : residue at $\mathrm{x}$ time 


\section{RESULTS AND DISCUSSION}

\section{Effect of certain environmental conditions on the fate of Finarmiol,} Triflumizole and penconazole formulations

\section{I.1. Storage stability at elevated temperature}

\section{Storage stability at $54 \pm 2^{\circ} \mathrm{C}$}

Table 1. Thermal stability of Finarimol, Triflumizole and penconazole during storage for different periods at $54^{\circ} \mathrm{C}$.

\begin{tabular}{|c|c|c|c|c|c|c|}
\hline \multirow{2}{*}{$\begin{array}{l}\text { Storage } \\
\text { period } \\
\text { (days) }\end{array}$} & \multicolumn{2}{|c|}{ Finarimol } & \multicolumn{2}{|c|}{ Triflumizole } & \multicolumn{2}{|c|}{ Penconazole } \\
\hline & $\begin{array}{c}\text { Active } \\
\text { ingredient }\end{array}$ & Loss $\%$ & $\begin{array}{c}\text { Active } \\
\text { ingredient }\end{array}$ & Loss $\%$ & $\begin{array}{c}\text { Active } \\
\text { ingredient }\end{array}$ & Loss $\%$ \\
\hline Initial & 12.00 & 0.00 & 15.00 & 0.00 & 10.00 & 0.00 \\
\hline 14 & 11.92 & 0.63 & 14.05 & 6.34 & 9.09 & 9.13 \\
\hline 21 & 11.61 & 3.22 & 14.01 & 6.62 & 9.07 & 9.26 \\
\hline 28 & 11.05 & 7.95 & 13.10 & 12.68 & 8.06 & 19.45 \\
\hline 45 & 10.39 & 13.40 & 13.09 & 12.75 & 6.09 & 39.06 \\
\hline 60 & 8.89 & 25.94 & 18.03 & 19.82 & 5.05 & 49.52 \\
\hline$R L_{50}$ & \multicolumn{2}{|c|}{28.4} & \multicolumn{2}{|c|}{18.73} & \multicolumn{2}{|c|}{5.74} \\
\hline
\end{tabular}

\section{Initial - 1 hour before storage}

\section{I.2. Effect of different temperatures}

Generally, data in tables (1-4) illustrate that Fenarimol was more stable than the other tested pesticides after storage for different periods at $54^{\circ} \mathrm{C}$ and the effect of different temperatures $\left(25^{\circ} \mathrm{C}, 35^{\circ} \mathrm{C}\right.$ and $\left.45^{\circ} \mathrm{C}\right)$. It is clearly evident that there is affirmative relationship between temperature and the rate of degradation. The previously mentioned results clearly showed that the rate of persistence of tested pesticides were influenced by many factors such as: chemical structures, vapor pressure, concentration of pesticides applied and period of exposure. In general increasing the period of exposure increased the rate of residues degradation. The statically half-lives for tested pesticides support that results. The results obtained agreed with the findings of Nasser (2001). 
Table 2. Effect of different temperature degrees on the persistence of Fenarimol residues.

\begin{tabular}{|c|c|c|c|c|c|c|}
\hline \multirow{2}{*}{$\begin{array}{c}\text { Time in } \\
\text { hours }\end{array}$} & \multicolumn{2}{|c|}{$2{ }^{\circ} \mathrm{C}$} & \multicolumn{2}{c|}{$3{ }^{\circ} \mathrm{C}$} & \multicolumn{2}{c|}{$45^{\circ} \mathrm{C}$} \\
\cline { 2 - 7 } Initial & 500.00 & 00.0 & 500.00 & - & 500.00 & - \\
\hline 1 & 499.95 & 0.01 & 484.90 & 3.02 & 482.14 & 3.57 \\
\hline 2 & 499.90 & 0.02 & 470.39 & 5.92 & 346.85 & 30.63 \\
\hline 4 & 499.80 & 0.04 & 445.25 & 10.50 & 287.32 & 42.54 \\
\hline 8 & 499.70 & 0.06 & 380.36 & 23.93 & 285.07 & 42.99 \\
\hline 12 & 497.75 & 0.45 & 363.94 & 27.21 & 280.04 & 43.99 \\
\hline 24 & 450.40 & 9.92 & 356.81 & 28.64 & 278.85 & 44.23 \\
\hline 48 & 392.30 & 21.54 & 334.65 & 33.07 & 252.55 & 49.49 \\
\hline 96 & 315.15 & 36.97 & 273.59 & 54.28 & 236.32 & 52.74 \\
\hline 192 & 178.90 & 64.22 & 202.78 & 59.44 & 175.53 & 64.89 \\
\hline$R L_{50}$ & & & & & & \\
\hline
\end{tabular}

Initial - 1 hour before storage

Table 3. Effect of different temperature degrees on the persistence of Trifilumizole residues.

\begin{tabular}{|c|c|c|c|c|c|c|}
\hline \multirow{2}{*}{$\begin{array}{c}\text { Time in } \\
\text { hours }\end{array}$} & \multicolumn{2}{|c|}{$25^{\circ} \mathrm{C}$} & \multicolumn{2}{c|}{$35^{\circ} \mathrm{C}$} & \multicolumn{2}{c|}{$45^{\circ} \mathrm{C}$} \\
\cline { 2 - 7 } & $\mu \mathrm{g}$ & Loss $\%$ & $\mu \mathrm{g}$ & Loss $\%$ & $\mu \mathrm{g}$ & Loss $\%$ \\
\hline Initial & 500.00 & 00.0 & 500.00 & - & 500.00 & - \\
\hline 1 & 499.22 & 0.16 & 499.06 & 0.19 & 494.68 & 1.06 \\
\hline 2 & 482.88 & 3.42 & 481.28 & 3.74 & 447.04 & 10.59 \\
\hline 4 & 473.77 & 5.25 & 406.23 & 18.75 & 305.94 & 38.81 \\
\hline 8 & 466.92 & 6.62 & 366.48 & 26.70 & 278.93 & 44.21 \\
\hline 12 & 454.14 & 9.17 & 357.95 & 28.41 & 273.46 & 45.31 \\
\hline 24 & 435.70 & 12.26 & 274.60 & 45.08 & 241.25 & 51.75 \\
\hline 48 & 414.78 & 17.04 & 242.89 & 51.42 & 238.63 & 52.27 \\
\hline 96 & 398.33 & 20.33 & 204.21 & 59.16 & 69.39 & 86.12 \\
\hline 192 & 189.44 & 62.11 & 129.09 & 74.18 & 62.65 & 87.47 \\
\hline$R L_{50}$ & & & \multicolumn{2}{|c|}{9.83} & & 5.7 \\
\hline
\end{tabular}


Table 4. Effect of different temperature degrees on the persistence of penconazole residues.

\begin{tabular}{|c|c|c|c|c|c|c|}
\hline \multirow{2}{*}{ Time in } & \multicolumn{2}{|c|}{$25^{\circ} \mathrm{C}$} & \multicolumn{2}{c|}{$35^{\circ} \mathrm{C}$} & \multicolumn{2}{c|}{$45^{\circ} \mathrm{C}$} \\
\cline { 2 - 7 } hours & $\mu \mathrm{g}$ & Loss $\%$ & $\mu \mathrm{g}$ & Loss $\%$ & $\mu \mathrm{g}$ & Loss \% \\
\hline Initial & 500.00 & 00.0 & 500.00 & - & 500.00 & - \\
\hline 1 & 496.33 & 0.73 & 495.7 & 0.86 & 481.35 & 3.73 \\
\hline 2 & 492.38 & 1.52 & 491.35 & 1.73 & 431.15 & 13.77 \\
\hline 4 & 479.99 & 4.00 & 482.2 & 3.56 & 373.85 & 25.23 \\
\hline 8 & 476.69 & 4.66 & 466.05 & 6.79 & 310.8 & 37.84 \\
\hline 12 & 436.95 & 12.61 & 426.6 & 14.68 & 190.55 & 61.89 \\
\hline 24 & 413.87 & 17.23 & 355.6 & 28.88 & 114.00 & 77.20 \\
\hline 48 & 331.54 & 33.69 & 227.7 & 54.46 & 50.4 & 89.92 \\
\hline 96 & 305.24 & 38.95 & 53.5 & 89.30 & 4.55 & 99.09 \\
\hline 192 & 245.40 & 50.92 & 22.2 & 95.56 & N.D. & - \\
\hline$R L_{50}$ & \multicolumn{2}{|c|}{23.34} & \multicolumn{2}{c}{8.05} & & 4.13 \\
\hline
\end{tabular}

Initial - 1 hour before storage

\section{I.3. Effect of ultraviolet rays (UV-rays) and direct sunlight}

The date presented in tables (5-6) and half-life statistically showed that the rate degradation of Penconazole was more rapid than Fenarimol and Triflumizole in their formulations, when exposed as dry films on glass surface to UV or sunlight .While Triflumizole was more stable

Table 5. Effect of UV-rays $(254 \mathrm{~nm})$ on the dissipation of Fenarimol, Triflumizole and Penconazole formulations.

\begin{tabular}{|c|c|c|c|c|c|c|}
\hline \multirow{2}{*}{$\begin{array}{c}\text { Time } \\
\text { exposure } \\
\text { in hours }\end{array}$} & \multicolumn{2}{|c|}{ Fenarimol } & \multicolumn{2}{c|}{ Triflumizole } & \multicolumn{2}{c|}{ Pencenazole } \\
\cline { 2 - 7 } & $\mu \mathrm{g}$ & Loss \% & $\mu \mathrm{g}$ & Loss \% & $\mu \mathrm{g}$ & Loss \% \\
\hline Initial & 500.00 & 00.0 & 500.00 & - & 500.00 & - \\
\hline 1 & 472.78 & 5.44 & 481.87 & 3.63 & 451.5 & 9.7 \\
\hline 2 & 439.20 & 12.16 & 479.07 & 4.19 & 444.35 & 11.13 \\
\hline 4 & 385.76 & 22.85 & 408.22 & 18.36 & 419.45 & 16.10 \\
\hline 6 & 324.98 & 35.00 & 387.21 & 22.56 & 393.8 & 21.24 \\
\hline 8 & 311.51 & 37.70 & 342.69 & 31.46 & 349.35 & 30.13 \\
\hline$R L_{50}$ & \multicolumn{2}{|c|}{12.31} & 15.67 & \multicolumn{2}{c|}{10.18} \\
\hline
\end{tabular}

Initial - 1 hour before storage 
Table 6. Effect of direct sunlight on pesticides used

\begin{tabular}{|c|c|c|c|c|c|c|}
\hline \multirow{2}{*}{$\begin{array}{c}\text { Time } \\
\text { exposure } \\
\text { in hours }\end{array}$} & \multicolumn{2}{|c|}{ Fenarimol } & \multicolumn{2}{c|}{ Triflumizole } & \multicolumn{2}{c|}{ Pencenazole } \\
\cline { 2 - 7 } & $\mu \mathrm{g}$ & Loss $\%$ & $\mu \mathrm{g}$ & Loss $\%$ & $\mu \mathrm{g}$ & Loss \% \\
\hline Initial & 500.00 & 00.0 & 500.00 & - & 500.00 & - \\
\hline 1 & 467.93 & 6.41 & 486.24 & 2.75 & 291.1 & 41.78 \\
\hline 2 & 395.66 & 20.87 & 404.57 & 19.09 & 278.35 & 44.33 \\
\hline 4 & 389.16 & 22.17 & 377.71 & 24.46 & 266.65 & 47.87 \\
\hline 6 & 330.00 & 33.46 & 358.35 & 28.33 & 220.6 & 55.88 \\
\hline 8 & 315.80 & 36.84 & 326.27 & 34.75 & 181.8 & 63.64 \\
\hline 12 & 296.04 & 40.79 & 320.23 & 35.95 & 148.1 & 70.38 \\
\hline 24 & 189.46 & 62.11 & 126.18 & 74.76 & 124.2 & 75.16 \\
\hline 48 & 162.15 & 67.57 & 118.86 & 76.23 & 55.05 & 88.99 \\
\hline 72 & 136.58 & 72.68 & 116.02 & 76.80 & 4.55 & 99.09 \\
\hline$R L_{50}$ & \multicolumn{2}{|c|}{4.42} & \multicolumn{2}{c}{5.52} & & 2.80 \\
\hline
\end{tabular}

\section{Initial - 1 hour before storage}

Triflumizole is photo chemically more stable than other tested pesticides which have imidazole ring cleavage as initial step of main pathway giving an $\mathrm{N}$-formyl degraded, 4-chloro- $\alpha, \alpha, \quad \alpha$-trifluoro- $\mathrm{N}$ - (1-formylamino-2-propoxyethilidene)-otoluidine, which is rapidly reformatted to a major 4-chloro- $\alpha, \alpha, \alpha$-trifluoro-N-(1-amino2pyopoxye thilidene)-o-toluidine followed by depropylation at the ether linkage. Hydrolysis products, such as 4-chloro- $\alpha, \alpha, \alpha$, trfiluoro-N-(propoxy acetyl)-O-toluidine and 4-chloro- $\alpha, \alpha, \alpha$, trifluoro-o-toluidine were also found as final products.( Hasselmann et al. (2008).

Penconazole is photo chemically unstable, and is readily broken by sunlight than other pesticides photo degradation of penconazole resulted in considerable formation of 1 -(4-chloro- $\beta$-propyl pheneathyl) $1-1 \mathrm{H}-1,2,4$-triazole (1) and $5 \mathrm{H}, 6 \mathrm{H}-$ (1,2,4-triazolo)-[5,1-a] -9- chloro-6- propyl iso quinoline (2). Furthermore, photodehalogenation of (2) yielded traces of $5 \mathrm{H}$, (1,2,4-triazolo)- [5, 1-a]-6-propylisoquinoline (3). On other hand, photo decomposition and photo dehalogenatin to photoproduct (1) were found to be main degradation pathways and photoproduct (2) was only detected as a trace component .(Wolfgang and Michael (1991).

The pesticide Fenarimol undergoes direct photo degradation, the main photo degradation pathway involves the hemolytic cleavage of the pyrimidine to the carbinol carbon bond. This primary reaction step is followed by fast in-cage recombination of 
the pyrimidine and ketyl radicals to form an intermediate that leads to a product (3) Mateus et al. (2000)<smiles>Cc1ccc(C(O)(c2ccc(Cl)cc2)c2cncnc2)c(Cl)c1</smiles>

Fenarimol

(1)<smiles>OC(c1ccc(Cl)cc1)c1cccc(Cl)c1-c1cncnc1</smiles>

(3)

Scheme (1): Main reaction pathways of Fenarimol

(According to Mateus et al,2000)

\section{Determination of physical properties}

\section{II.1) Density properties}

The data presented in table (7), indicated that all the tested pesticides: had justified results.

Table 7. Effect of storage temperature on density of tested pesticides.

\begin{tabular}{|c|c|c|c|c|}
\hline $\begin{array}{c}\text { Storage } \\
\text { temperature }\end{array}$ & $\begin{array}{c}\text { Storage } \\
\text { period (days) }\end{array}$ & $\begin{array}{c}\text { Rubigan } \\
(12 \%) \mathrm{g} / \mathrm{cm}^{3}\end{array}$ & $\begin{array}{c}\text { Trifimine } \\
(15 \%) \mathrm{g} / \mathrm{cm}^{3}\end{array}$ & $\begin{array}{c}\text { Topas } \\
(10 \%) \mathrm{g} / \mathrm{cm}^{3}\end{array}$ \\
\hline \multirow{7}{*}{$54^{\circ} \mathrm{C}$} & Initial & 0.99163 & 0.931 & 0.9714 \\
\cline { 2 - 5 } & 14 & 1.01303 & 0.95 & 0.98914 \\
\cline { 2 - 5 } & 21 & 1.0130 & 0.954 & 0.98885 \\
\cline { 2 - 5 } & 28 & 1.0130 & 0.954 & 0.99715 \\
\cline { 2 - 5 } & 45 & 1.0130 & 0.954 & 0.997 \\
\cline { 2 - 5 } & 60 & 1.0128 & 1.0154 & 0.997 \\
\hline
\end{tabular}

Initial = one hour before storage

\section{II.2) Alkalinity or acidity}

The data presented in table (8), indicated that all tested pesticides, reveal high acidity through different periods of storage. These results are in agreement with the finding of Abd El-All et al. (1993 a). 
Table 8. Effect of storage temperature on free acidity\% as $\mathrm{H}_{2} \mathrm{SO}_{4}$ for tested pesticides.

\begin{tabular}{|c|c|c|c|c|}
\hline $\begin{array}{c}\text { Storage } \\
\text { temperature }\end{array}$ & $\begin{array}{c}\text { Storage period } \\
\text { (days) }\end{array}$ & $\begin{array}{c}\text { Rubigan } \\
(12 \%) \mathrm{g} / \mathrm{kg}\end{array}$ & $\begin{array}{c}\text { Trifimine } \\
(15 \%) \mathrm{g} / \mathrm{kg}\end{array}$ & $\begin{array}{c}\text { Topas } \\
(10 \%) \mathrm{g} / \mathrm{kg}\end{array}$ \\
\hline \multirow{7}{*}{$54^{\circ} \mathrm{C}$} & Initial & 0.293 & 0.245 & 0.637 \\
\cline { 2 - 5 } & 14 & 0.989 & 0.294 & 0.8134 \\
\cline { 2 - 5 } & 21 & 0.98 & 0.3234 & 2.058 \\
\cline { 2 - 5 } & 28 & 1.421 & 0.5782 & 4.802 \\
\cline { 2 - 5 } & 45 & 1.421 & 0.5782 & 5.243 \\
\cline { 2 - 5 } & 60 & 1.519 & 0.549 & 5.439 \\
\hline
\end{tabular}

Initial $=$ one hour before storage

\section{II.3) $\mathrm{pH}$ range}

The data presented in table (9)indicated that data of all tested pesticides, agreed with FAO/ WHO Manual (2002, 2006), El Attal and El-sisi (1979).

Table 9. Effect of storage temperature on $\mathrm{pH}$ values for tested pesticides.

\begin{tabular}{|c|c|c|c|c|}
\hline $\begin{array}{c}\text { Storage } \\
\text { temperature }\end{array}$ & $\begin{array}{c}\text { Storage period } \\
\text { (days) }\end{array}$ & $\begin{array}{c}\text { Rubigan } \\
(12 \%)\end{array}$ & $\begin{array}{c}\text { Trifimine } \\
(15 \%)\end{array}$ & $\begin{array}{c}\text { Topas } \\
(10 \%)\end{array}$ \\
\hline \multirow{5}{*}{$54^{\circ} \mathrm{C}$} & Initial & 6.33 & 6.43 & 5.87 \\
\cline { 2 - 5 } & 14 & 5.45 & 6.33 & 5.71 \\
\cline { 2 - 5 } & 21 & 5.40 & 6.20 & 4.88 \\
\cline { 2 - 5 } & 28 & 4.98 & 6.14 & 3.68 \\
\cline { 2 - 5 } & 45 & 4.98 & 6.14 & 3.68 \\
\cline { 2 - 5 } & 60 & 4.2 & 6.00 & 3.35 \\
\hline
\end{tabular}

\section{Initial $=$ one hour before storage}

\section{II.4) Emulsion stability}

The data presented in table (10), indicated that for all tested pesticides, Emulsion stability after 30 minutes under soft and hard water has no sedimentation and agree with specification WHO (1979). 
Table 10. Effect of storage temperature on free Emulsion stability for tested pesticides.

\begin{tabular}{|c|c|c|c|c|}
\hline $\begin{array}{c}\text { Storage } \\
\text { temperature }\end{array}$ & $\begin{array}{c}\text { Storage period } \\
\text { (days) }\end{array}$ & $\begin{array}{c}\text { Rubigan } \\
(12 \%)\end{array}$ & $\begin{array}{c}\text { Trifimine } \\
(15 \%)\end{array}$ & $\begin{array}{l}\text { Topas } \\
(10 \%) \\
\end{array}$ \\
\hline \multirow{6}{*}{$54^{\circ} \mathrm{C}$} & Initial & $\begin{array}{c}\text { Acceptable } \\
\text { Limit }\end{array}$ & $\begin{array}{c}\text { Acceptable } \\
\text { Limit }\end{array}$ & $\begin{array}{c}\text { Acceptable } \\
\text { Limit }\end{array}$ \\
\hline & 14 & $\begin{array}{c}\text { Acceptable } \\
\text { Limit }\end{array}$ & $\begin{array}{c}\text { Acceptable } \\
\text { Limit }\end{array}$ & $\begin{array}{c}\text { Acceptable } \\
\text { Limit }\end{array}$ \\
\hline & 21 & $\begin{array}{c}\text { Acceptable } \\
\text { Limit }\end{array}$ & $\begin{array}{c}\text { Acceptable } \\
\text { Limit } \\
\end{array}$ & $\begin{array}{c}\text { Acceptable } \\
\text { Limit } \\
\end{array}$ \\
\hline & 28 & $\begin{array}{c}\text { Acceptable } \\
\text { Limit }\end{array}$ & $\begin{array}{c}\text { Acceptable } \\
\text { Limit } \\
\end{array}$ & $\begin{array}{c}\text { Acceptable } \\
\text { Limit } \\
\end{array}$ \\
\hline & 45 & $\begin{array}{c}\text { Acceptable } \\
\text { Limit }\end{array}$ & $\begin{array}{c}\text { Acceptable } \\
\text { Limit }\end{array}$ & $\begin{array}{c}\text { Acceptable } \\
\text { Limit }\end{array}$ \\
\hline & 60 & $\begin{array}{c}\text { Acceptable } \\
\text { Limit }\end{array}$ & $\begin{array}{c}\text { Acceptable } \\
\text { Limit } \\
\end{array}$ & $\begin{array}{c}\text { Acceptable } \\
\text { Limit }\end{array}$ \\
\hline
\end{tabular}

Initial $=$ one hour before storage

\section{REFERENCES}

1. Abd-El-All Farage, A.G. Sisi, M.A. El-Hamaky and A. Affity. 1993a. effect of storage conditions and chemical stability of formulations containing pesticides Mixtures. Egypt. J. Appl. Sci. 8(10): 16-23.

2. Attenburger, E. 1995b. Penconazole CCGA 71818, JMRR 1995. Letter from CibaGeigy Itd., Basle, 08, June 1995, unpublished.

3. CIPACF. 1995. Physico-chemical methods for technical and formulated pesticides, V.F, collaborative international pesticides analytical council limited. MT3P 11-19, MT 31 p 96-102, MT 36 p. 108-114, MT 75, p. 205-206.

4. El-Attal, Z. M. and A. G. Elsisi. 1979. The role of chelating agents and polar solvents pesticides formulation in hard water. Bullent, Soc. Egypt Econ. Ser 11,P193-199.

5. FAO specifications. 1988. Insecticides Emulsifiable concentrates storage stability $54 \pm 2{ }^{\circ} \mathrm{C}$ for 14 days $\mathrm{p} 19,20$.

6. FAO/WHO Manual. 2002 and 2006, Manual on development and use of FAO and WHO specifications for pesticides first Edition. P50, 51, p. 120, 121. March 2006 revision of the first edition P 58, 59, 60, p. 125, 127. 
7. Hasselmann C., C. Pigoult, R. Santust, G laustriat. 2008. Photo-degradation of the imidazole ring induced by indole driva oxygen-free aqueous Solutions photochemistry and photobiology volume 27 issue 1, pages 13-18.

8. Mateus M. C. D. A., A. M. Silva, H. Burrows. 2000. Kinetics of photo degradation of the fungicide Fenarimol in natural waters and in various salts solutions: salinity effects and mechanistic consideration. Water Research 34, 1119-11126.

9. Morse lab. 1989. Uniroy363al a chemical company Inc., Analytical Method for the determination of Triflumizole Residues in crops volume 6 pp \# 6F3372/FAP\# $6 \mathrm{H} 5497$.

10. Moye, H. A., M. A. Malagodi, G. L. Jyoh, C. C. K. Leibea and P. G. Wislocki. 1987. Residues of avermectin $B_{10}$, rotational crop and soils following soil treatment with $\mathrm{C}_{14}$ avermectin $\mathrm{B}_{1 \mathrm{a}}$ J. Agric food chem.. 35, 859-864.

11. Nasser, IN. 2001. Fate of some pesticides and environmental conditions. Thesis, Degree of doctor of philosophy in Agricultural sciences (pesticides).

12. Samtoro, A. A. scopa, S. A. Bufo, M. Mansour, H. Mountacer. 2000. Photo degradation of the triazole fungicide Hexaconazole Bull. Environm. Environmental Contamination Toxicology. Contam. Toxicol 64, 475-480.

13. Tomlin C. (Ed). 1994. The pesticides Menual, 10th ed., British crop protection council and Royal society of chemistry, Cambridge.

14. WHO. 1979. Specification for pesticide used in public health p. 116, Geneva, Switzerland.

15. Wolfgang, S. and H. Michael. 1991. Fungicides and photochemistry photo degradation of Azole fungicide Penconazole. Zeitschrift tur lebenemittel untersuchung und forschung: 198: 11-14. 


\section{ثبات الفيناريمول والتراى فليمازول والبيناكونارول فى مستحضراتها التجارية تحت بعض الظروف البيئية المختلفة}

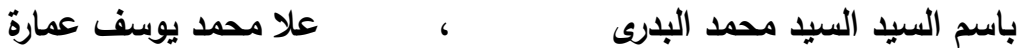

قسم بحوث تحليل المبيد/ت - الدعهل المركزى للمبيدات - مركز البحوث الزراعية - الدقى - الجيزة

هذا البحث يلقى الضوء على دراسة ثبات الفيناريمول (الروبيجان r ا\%)، والتراى فليوميزول

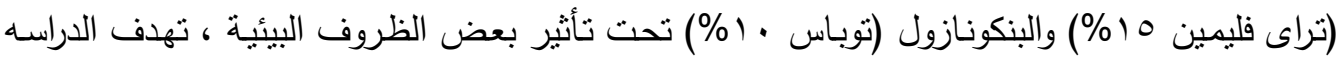

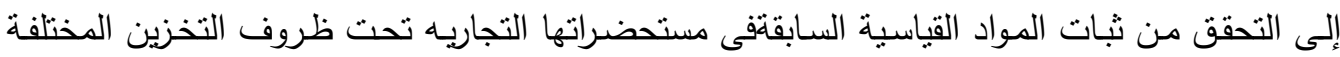

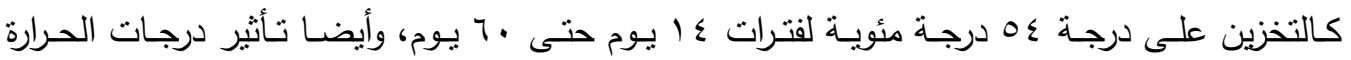

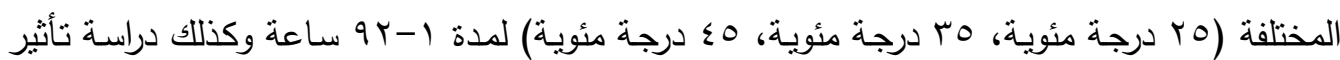

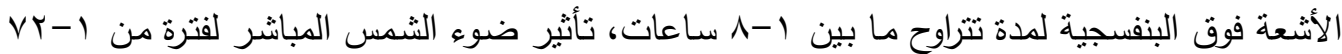
ساعة، توضح هذه الدراسة نتائج الخواص الطبيعية المناسبة على المبيدات المختبرة قبل وبعد التخزين.

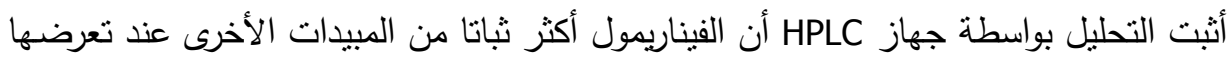
لظروف التخزين المختلفة تحت درجات الحرارة المختلفة. هناك علاقة طردية بين درجات الحرارة وبين

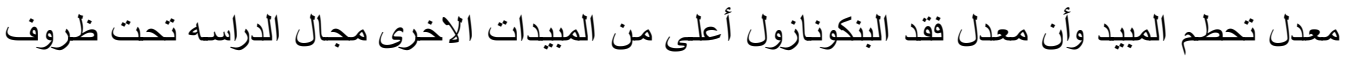
حراريه 0Y درجة مئوية ، 0ب درجة مئوية، 0 ـ درجة مئوية.

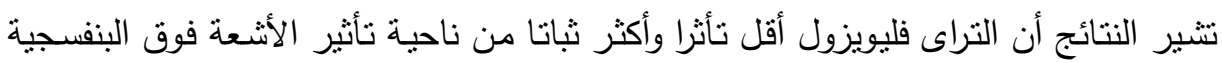

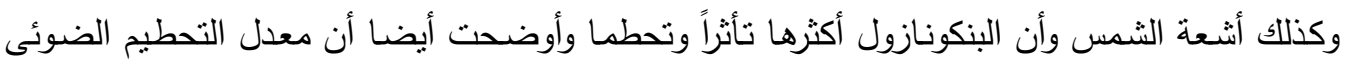
يزداد بزيادة فترة التعريض. كانت نتائج اختبارات الخواص الطبيعيـة للمبيدات المختبرة قبل وبعد التخزين في الحدود الدولية المسموح بها طبقا للخطوط الإرشادية لمنظمة الأغذية والزراعة (FAO) ومنظمة الصحة العالمية 